\title{
Antimicrobial resistance. Its importance and efforts to control it
}

\author{
Silvia Giono-Cerezo, ${ }^{1 *}$ José I. Santos-Preciado, ${ }^{2}$ María del Rayo Morfín-Otero, ${ }^{2}$ \\ Francisco J. Torres-López ${ }^{3}$ and María Dolores Alcántar-Curiel${ }^{4}$
}

${ }^{1}$ Instituto Politécnico Nacional, National School of Biological Sciences, Department of Microbiology, Mexico City; ${ }^{2}$ Universidad de Guadalajara, Hospital Civil de Guadalajara "Fray Antonio Alcalde", Guadalajara, Jalisco; ${ }^{3}$ nstituto Mexicano del Seguro Social, Centro Médico Nacional Siglo XXI, Pediatric Hospital, Mexico City; ${ }^{4}$ Universidad Nacional Autónoma de México, Faculty of Medicine, Experimental Medicine Unit, Mexico City. Mexico

\begin{abstract}
The World Health Organization estimates that bacterial resistance will cause 10 million deaths by 2050. As part of the Global Action Plan on Antimicrobial Resistance, it proposed networks of specialized laboratories in order to preserve strains and optimize the use of antimicrobials. That is the case of the Latin American Surveillance Network of Antimicrobials Resistance. In a 2019 study, the main bacteria of the ESKAPE group (which are highly resistant to the most widely used antibiotics) that cause infections in Mexican Hospitals were identified to be multidrug-resistant (MDR) and extended-spectrum beta-lactamase (ESBL)-producing Klebsiella spp., ESBL-producing Enterobacter spp., Acinetobacter baumannii, MDR Pseudomonas aeruginosa, methicillin-resistant Staphylococcus aureus and vancomycin-resistant Enterococcus faecium. With information on drug resistance, regimens are recommended to treat infection caused by Helicobacter pylori, a pathogen related to the development of cancer and whose prevalence in the adult population of Latin America is estimated to range between 60 and $70 \%$.
\end{abstract}

KEY WORDS: National Strategy. Antimicrobial resistance. ESKAPE group. Helicobacter pylori.

\section{Resistencia antimicrobiana. Importancia y esfuerzos por contenerla}

\section{Resumen}

La Organización Mundial de la Salud estima que en 2050 la resistencia bacteriana ocasionará 10 millones de muertes. Como parte del Plan de Acción Mundial sobre la Resistencia a los Antimicrobianos propuso redes de laboratorios especializados, para conservar cepas y optimizar el uso de los antimicrobianos. En un estudio de 2019 se identificó que las principales bacterias del grupo ESKAPE (con alta resistencia a los antibióticos más usados) que causan infecciones en hospitales de México son Klebsiella spp. resistentes a múltiples fármacos (MDR) y productoras de betalactamasa de espectro extendido (BLEE), Enterobacter spp. BLEE, Acinetobacter baumannii, Pseudomonas aeruginosa MDR, Staphylococcus aureus meticilinorresistente y Enterococcus faecium resistente a vancomicina. Con la información de resistencia a los fármacos se recomiendan esquemas para tratar la infección causada por Helicobacter pylori, relacionado con el desarrollo de cáncer y cuya prevalencia en la población adulta de Latinoamérica se estima es de entre 60 y $70 \%$.

PALABRAS CLAVE: Estrategia Nacional. Resistencia antimicrobiana. Grupo ESKAPE. Helicobacter pylori.

\footnotetext{
Correspondence:

Date of reception: 12-11-2019

*Silvia Giono-Cerezo

Date of acceptance: 03-01-2020

E-mail: sgiono@yahoo.com

DOI: 10.24875/GMM.M20000358

Gac Med Mex. 2020;156:171-178

Contents available at PubMed license (http://creativecommons.org/licenses/by-nc-nd/4.0/).
} 


\section{Introduction}

Antimicrobial resistance is defined as the ability of a microorganism to resist the effects of antibiotics; it is an inherent characteristic of bacteria or it can be an ability that is acquired during the infectious process.

According to the United Nations (UN), bacterial resistance is one of the main health threats, as it endangers global priorities such as human development. In view of the profound implications in economic activities, food supply, tourism and migration flows, based on this redefinition, cooperation, consultation and surveillance mechanisms have been established with more or less support from each country.

There are three types of infections according to the resistance of the bacteria that cause them:

- Enterobacteriaceae infections, due to their impact on morbidity and mortality.

- Acinetobacter spp. infections. Reports in several hospitals reveal that there are limited therapeutic options with available antibiotics. Antibiotics generated in recent years also have no activity against these multi-drug resistant (MDR), extensively drug resistant (XDR) or pandrug resistant (PDR) strains.

- Other serious infections, such as those caused by Pseudomonas aeruginosa, which can cause high mortality.

The motto of the World Health Organization (WHO) regarding resistance to antimicrobials is "no action today, no cure tomorrow", since it is estimated that bacterial resistance will cause 10 million deaths per year by 2050, and a reduction of between 2 and $5 \%$ of gross domestic product in some countries. Based on its risk report, during the 2013 World Economic Forum, this health problem was positioned at the same level as the risk of weapons of mass destruction proliferation and global economic crisis. ${ }^{1}$

The 17 sustainable development goals of the UN 2030 agenda are a call to action to all countries to eradicate poverty and protect the planet, as well as to guarantee peace and prosperity; seven of these objectives have to do with antimicrobial resistance. ${ }^{2}$

In the 2015 Global Action Plan, the Global Surveillance System was established, where there was a resolution by the WHO assemblymen/women group on antimicrobial resistance, with the idea of presenting the countries' advances in the $72^{\text {nd }}$ World Health Assembly, in May 2019; unfortunately, neither the
Mexican Minister of health nor the undersecretary were able to attend in order to present the country's advances. ${ }^{3}$

In the Global Action Plan there are five objectives related to the strengthening of knowledge based on surveillance and research studies.

With regard to global actions, countries have different methodologies to address the problems. An exemplary case is that of the United States, a country that has joined the Global Antimicrobial Resistance Surveillance System (GLASS), which refers to the use of surveillance standards whereby improving patient safety is intended. The need to ensure data quality and standardize the reports on resistant bacteria stands out. ${ }^{4}$

In the United States, the Centers for Disease Control and Prevention (CDC) analyze healthcare-associated infections (HAls) through a national network that has more than 24,000 health facilities, as well as more than 63 thousand individual users, which gives certainty about the quality of the generated data. ${ }^{5}$

The WHO collects and analyzes data on antimicrobial resistance and adds them to information related to the countries following standard definitions; in 2011, it dedicated World Health Day to the fight against antimicrobial resistance; in 2015, it recommended member countries to develop a national plan to fight antimicrobial resistance; in 2017, it launched the Global Antimicrobial Resistance Surveillance Program.

In 2017, the National Strategy for Action against Antimicrobial Resistance was announced in Mexico, which was published as Action plan against antimicrobial resistance for Mexico and whose working group includes the Ministry of Public Education, the Ministry of Health, the Ministry of Agriculture and Rural Development, the Ministry of Environment and Natural Resources, the Ministry of Economy, the Ministry of Treasury and Public Credit, the Ministry of Foreign Affairs, the Mexican Institute of Social Security and the Institute of Social Security and Services of State Workers, under the coordination of the Federal Commission for Protection against Health Risks. With this multi-sectoral strategy, epidemiological and health surveillance, the use of antimicrobials in human health and research are aligned. ${ }^{6}$

On June 5, 2018, an agreement was published in the Official Gazette of the Federation declaring the mandatory adherence to the National Strategy for Action against Antimicrobial Resistance by all the institutions that make up the National Health System. ${ }^{7}$ 


\section{Bacterial resistance regional networks}

Research on microbial resistance to antibiotics is conducted in interdisciplinary laboratory networks and in national, regional and international research associations. Interdisciplinary approaches are generally useful, even indispensable, for a successful investigation of complex problems such as bacterial resistance. The networks generate information regarding affected subjects, in which hospitals, to which magnitude and extent, what types of antibiotics are used, impact on mortality, severity of the disease and economic impact, among other data. The best definition is that microbial resistance surveillance networks favor better actions for prevention and control.

\section{Latin American Network for Antimicrobial Resistance Surveillance (ReLAVRA)}

The Latin American Network for Antimicrobial Resistance Surveillance (ReLAVRA - Red Latinoamericana de Vigilancia de la Resistencia a los Antimicrobianos), created in 1996 with support from the Pan American Health Organization, has the mission to obtain reliable, timely and reproducible microbiological data to be used in the improvement of patient care through the establishment of sustainable quality assurance programs. ${ }^{8}$

The first network in Mexico was that of the Mexican Association of Infectology and Clinical Microbiology, which operated from 1997 to 2000, and worked in a quality laboratory system for the study of gram-positive and negative bacteria. Then, the UME Hospital Network followed. In turn, the National Autonomous University of Mexico, through the University Program for Health Research, established the University Plan for the Control of Antimicrobial Resistance. ${ }^{9}$

The Thematic Network for Drug Resistance Research and Surveillance is perhaps the most ambitious and productive, since it includes 50 hospitals in 20 different states. ${ }^{10,11}$

ReLAVRA includes four countries: Argentina, Brazil, Mexico and Spain; the latter involves the participation of the University of Madrid and the Complutense University. This research network allows the exchange of knowledge and learning between postgraduate students and researchers.

The reduced number of networks based on specific pathologies and with indicators obtained from the comparison of community-acquired infections versus HAls and other targets limits the construction of denominators to know the real impact of antimicrobial resistance. The WHO and the Pan American Health Organization have made an effort in the region of the Americas to define guidelines and convene member countries to work in the development of surveillance plans. The guidelines indicate basic elements for implementing and adapting surveillance networks according to the capacities and development of the region (GLASS). ${ }^{4}$

In Mexico, national antimicrobial resistance surveillance programs should go from being expository to being evolutionary and guarantee the quality of information, which must be public and transparent in order to adequately guide actions at the local and regional levels and be accessible to international agencies.

\section{WHO recommendations on antimicrobial resistance in HAls}

Seven-hundred thousand cases of antimicrobial resistance have been recorded, which is more than those of cancer, cholera, diabetes and diarrhea. Due to its impact, antimicrobial resistance requires an investment of 2 to $3.5 \%$ of gross domestic product; if this is not carried out by 2050 , estimates indicate that there will be more than 10 million deaths per year.

Antimicrobial resistance can be originated by two mechanisms:

- Artificial selection due to inadequate antibiotic treatment, in which resistant clones are selected.

- Natural selection, which refers to horizontal transfer of genes, where there is an acquisition of plasmids with resistance genes, which increases the prevalence of resistant bacteria. ${ }^{12}$

In the United States, the CDCs point out the need to early prevent and detect bacterial resistance. Identification, control, monitoring and surveillance are the only actions that can help prevent the spread of antimicrobial resistance.

Although there are examples on how antimicrobial resistance spreads in the environment, in the community and in hospitals, it is necessary for research to delve into either of these areas, as well as to manage and know what is going on at those levels. ${ }^{13}$

Reports indicate that there is antimicrobial resistance to new antibiotics that are launched onto the market, with an example among many being linezolid, which started being marketed in 2000 and by 2001 there were already reports of resistance..$^{14}$

In 2017, the WHO promoted the investigation and development of antibiotics that are no longer produced 
by the pharmaceutical industry; furthermore, it pointed out that there are microorganisms with critical priorities because they cause infections with high morbidity and mortality, such as Acinetobacter baumannii, Pseudomonas aeruginosa, Enterococcus, Helicobacter pylori, Salmonella, Campylobacter and Neisseria gonorrhoeae (responsible for cephalosporin- and fluoroquinolone-resistant sexually transmitted infection); as medium priority was left Streptococcus pneumoniae, for which establishing its resistance profile is necessary, in addition to Hemophilus influenzae and Shigella.

One suggestion is to stratify bacteria as MDR, XDR and PDR, as it is done for Mycobacterium tuberculosis, since there are increasingly frequent reports of strains with these characteristics.

The WHO proposes an action plan based on raising awareness on the problem, strengthening knowledge and reducing the incidence of infections through preventive measures such as environmental sanitation, hand washing and optimal use of antimicrobials, both in humans and animals. ${ }^{5}$ The action plan includes improving knowledge and understanding of antimicrobial resistance in local and national settings, surveillance of its incidence, optimization of the group of antibiotics and development of containment measures. Knowledge about the pharmacodynamics and pharmacokinetics of these antimicrobials, the mechanisms of action of antibiotics and antimicrobial resistance and transmission, as well as the assessment of the magnitude of prevalence depends on microbiologists and doctors.

The microbiologist can collaborate in the training of health personnel and carry out campaigns of awareness and understanding of the problem by participating in undergraduate education, as well as by strengthening knowledge through surveillance and research. In the laboratory, microbiologists can investigate the mechanisms of resistance, and detect, confirm and measure the impact of antimicrobial resistance, as well as provide microbiological knowledge, and thereby support the doctor in the development of treatment guidelines based on local epidemiological studies in human beings, animals and the environment. ${ }^{15}$

Information users should know the different strategies, which consist of:

- Strengthening knowledge in order to make a good use of information, both locally and nationally.

- Measuring the regional trend of antimicrobial resistance.

- Prioritizing strategies, and formulating a consensus and global recommendations.
- Determining the components of the surveillance system and the type of samples arriving to laboratories.

- Characterizing the antimicrobial sensitivity profile, ${ }^{6}$ with internal and external protocols and quality controls.

- Submitting reports and ensuring that they are accepted and applied in hospitals or in the community, as appropriate.

Many of these measures are already regulated; for example, antibiotic sensitivity tests are carried out in laboratories according to the Clinical and Laboratory Standards Institute (CLSI), which updates its standards and guidelines on an annual basis.

There are levels of surveillance that can be prioritized and that can serve to determine which the empirical treatment should be, when to apply hospital or individual programs (if they exist) regarding the use of antibiotics and infection control.

ReLAVRA supports the WHO criteria to determine what does occur at each country of the region. In order to reduce the incidence of infections, effective hygiene and prevention and control measures are recommended, such as effective hand hygiene, cleaning procedures, reduction of healthcare-associated infections, vaccination, and environmental sanitation; regarding animal health, biosafety and hygiene and sustainable animal production are also indicated.

Regarding the above, microbiologists can collaborate with proper management and identification of clinical samples, with the development of appropriate cultures and the performance of accurate and timely sensitivity tests, with the surveillance of patients and the environment, when required. With regard to early detection of events that can turn into outbreaks in hospitals and in the community, they can collaborate with microbiological data, characterization of outbreaks, evaluation of the spread of nosocomial and community pathogens, assessment of the impact of prevention strategies (vaccines) and alerting health personnel on the emergence of antimicrobial resistance in pathogens already under surveillance or on emerging pathogens. ${ }^{8}$

The Network must insist on the best use of antimicrobials, on timely and adequate diagnosis and on reporting according to the place of infection, with special surveillance tests, as well as on the custody of strains.

The CLSI formulated some rapid, complementary and confirmatory tests that can help guide the doctor for evaluation or alternative treatments. 
Carbapenem-resistant enterobacteriaceae (CRE) global spread started in North Carolina, and from there it disseminated to New York, Israel and Europe; carbapenemase-producing CREs of the New Delhi metallo- $\beta$-lactamase (NDM) type originated in India, and from there they spread to several countries. ${ }^{16}$

As for the animal population (fish, domestic fowl and pets), studies are needed to assess environmental resistance..$^{17}$

In order to comply with the Global Action Plan on Antimicrobial Resistance, it is necessary to ensure the sustainability of evaluation through research, which requires investment and the establishment of procedures, as well as accepting the role of the laboratory, which will impact on patient benefit.

\section{ESKAPE group in Mexico}

Antimicrobial resistance is a worldwide public health problem related to human and non-human antimicrobials use; it is one of the subjects addressed in the multisectoral approach called 'One Health', which brings the WHO, the United Nations Food and Agriculture Organization and the World Organization for Animal Health together. ${ }^{18}$

Currently, the most serious infections that threaten human life are caused by a group of antibiotic-resistant bacteria, which the American Society for Infectious Diseases has named the ESKAPE group. ESKAPE is an acronym formed by the name of six bacteria that cause serious infectious diseases and whose pathogenicity and antimicrobial resistance mechanisms are evolutionarily highly developed. ${ }^{19}$ ESKAPE group bacteria are a critical health threat because they cause a substantial percentage of HAls in modern hospitals (the CDCs indicate that they are responsible for two thirds of HAls), represent the majority of isolates whose resistance to antibiotics is severe and are pathogenesis, transmission and resistance paradigms, and therefore drive the doctor to therapeutic dilemmas.

The bacteria of the ESKAPE group are the following:

1. Vancomycin-resistant enterococcus faecium has emerged as a nosocomial pathogen that causes urinary tract, wound and bloodstream infections, and has also been linked to infections deriving from the use of catheters and surgical procedures.

2. Staphylococcus aureus is part of the microbiota of the skin and moist areas of the human body. In Mexican carriers, strains with variable antimicrobial susceptibility have been identified. With regard to HAls, S. aureus has been associated with bacteremia, surgical wound infections, endocarditis, pyogenic arthritis, osteomyelitis, and skin and soft tissue infections. In isolates, it exhibits resistance to antibiotics and beta-lactams, including methicillin, in which case it is called MRSA (methicillin-resistant Staphylococcus aureus).

3. Klebsiella pneumonae is an enterobacterium that represents a level of urgent threat; it causes respiratory, urinary and bloodstream infections, acquired both in hospitals and in the community. In hospitals, where this pathogens can spread and cause outbreaks, extended-spectrum $\beta$-lactamase (ESBL)-producing strains have been isolated, including carbapenemase-producing strains.

4. Acinetobacter baumannii is currently recognized as a pathogen causative of infections in patients admitted to the intensive care unit; it causes pneumonia and bacteremia associated with the use of catheters. At present, most isolates show multidrug resistance, even to carbapenems.

5. Pseudomonas aeruginosa is an opportunistic nosocomial pathogen that causes pneumonia, bacteremia, urinary tract and surgical wound infections, which is also found in the environment. The number of infections caused by this bacterium has increased and it is mostly MDR.

6. Enterobacter cloacae is a bacillus that is present in the digestive tract. It has been associated with urinary tract and surgical wound infections and bacteremia, but more often it has been identified in hospitalized immunocompromised patients. Due to the low permeability of its outer membrane, enterobacteria exhibit resistance to penicillin, oxazoyl penicillin, clindamycin, lincomycins, glycopeptides (vancomycin and teicoplanin) and macrolides.

The first study on ESKAPE group bacteria surveillance in Mexico was published in 2012, and was conducted over the course of one year at the Intensive Care Unit of the University Hospital in Monterrey, Nuevo León. A total of 1693 pathogens from different clinical samples were analyzed; ESKAPE group bacteria were found to account for $64 \%$ of isolates. $A$. baumannii was found to be the most common, followed by $P$. aeruginosa. ${ }^{20} P$. aeruginosa and $A$. baumannii were MDR, even to carbapenems; $20 \%$ of $A$. baumannii isolates were PDR; $36 \%$ of MDR K. pneumoniae isolates were of the extended-spectrum $\beta$-lactamase-producing type. The resulting infections had few antimicrobial treatment options. Regarding gram-positive bacteria, $62 \%$ of $S$. aureus isolates 
showed resistance to methicillin and $4 \%$ to vancomycin; as for Enterococcus spp., $10 \%$ were observed to be resistant to vancomycin.

The second work published in Mexico was carried out at the National Cancer Institute. ${ }^{21}$ The ESKAPE group bacteria isolated from blood cultures of cancer patients were analyzed. More than 33 thousand blood cultures obtained over a 10-year period were analyzed. In $17 \%$ of the blood cultures there was bacterial isolation, with $92 \%$ of them being MDR and $58 \%$ gram-negative bacilli; $6 \%$ were K. pneumoniae, out of which $11 \%$ were ESBL-producing; $P$. aeruginosa was identified in $6 \%$, out of which $11 \%$ were MDR. E. cloacae was found with a high BLEE production percentage, as well as $A$. baumannii, with $24 \%$ being MDR. Gram-positive pathogens showed $37 \%$ of MDR, $9 \%$ were $S$. aureus, $21 \%$ were resistant to methicillin, $2 \%$ were Enterococcus spp., and $32 \%$ were resistant to vancomycin. The researchers concluded that MDR strains of the ESKAPE group were the most frequently isolated strains in patients with hematologic malignancies. ${ }^{21}$

The third report was published by the University Program of Health Research of the National Autonomous University of Mexico, and it shows antimicrobial resistance current status in Mexico. A total of 11,900 isolates obtained during 2016 and 2017 from 14 hospitals of six states of the Mexican Republic were included. The majority of isolates (73\%) came from urine cultures; Escherichia coli was identified in $91 \%$, and K. pneumoniae in $8.5 \%$; $27 \%$ of isolates were obtained from blood cultures. Gram-negative bacteria were E. coli, K. pneumoniae, E. cloacae, $P$. aeruginosa and $A$. baumannii. K. pneumoniae and E. cloacae showed resistance to all cephalosporins and $60 \%$ of the isolates were ESBL producers, which clearly shows the urgency of interventions to control the problem of antimicrobial resistance. The gram-positive isolates studied were $S$. aureus, which accounted for $21 \%{ }^{9}$

The most recent work, in which 47 hospitals from 20 states of Mexico participated, was published in March 2019. A total of 22,943 isolates obtained from January to June 2018 were studied, with a high percentage of carbapenemase-resistant gram-negative bacteria being found: more than $50 \%$ of $A$. baumannii, $40 \%$ of $P$. aeruginosa and $12 \%$ of Klebsiella spp. and $E$. cloacae. MDR was quite high in $A$. baumannii $(53 \%)$ and $K$. pneumoniae (22 \%). In the group of gram-positive bacteria, $21 \%$ were methicillin-resistant S. aureus and $21 \%$ were vancomycin-resistant enterococci. ${ }^{10}$
In summary, the main bacteria of the ESKAPE group that cause nosocomial infections in Mexico were MDR ESBL-producing Klebsiella spp., ESBL-producing Enterobacter spp., MDR A. baumannii and $P$. aeruginosa (both resistant even to carbapenems), methicillin-resistant $S$. aureus and vancomycin-resistant E. faecium.

In recent years, infections by ESKAPE group bacteria have increased in Mexico, mostly in intensive care units; isolates have been characterized as MDR. MDR $A$. baumannii and MDR K. pneumoniae require immediate attention, as well as vancomycin-resistant MRSA and E. faecium strains. It is important to consider that these isolates are the starting point for outbreaks of infections related to healthcare-associated bacteremia.

Finally, it is necessary to update and maximize the use of antimicrobial treatments, in order to preserve their usefulness. Information from studies conducted in Mexico points at the urgency of implementing measures to control antimicrobial resistance. If we don't act today, there will be no cure tomorrow.

\section{Antibiotics can prevent Helicobacter pylori-associated gastric cancer}

Gastric cancer is the second cause of mortality due to tumors in the world. Particularly in developing countries, treatment success depends on early diagnosis; unfortunately, this cancer alerts the patient too late to seek medical help. ${ }^{22}$

The Global Cancer Observatory (GCO) points out areas where gastric cancer rates are higher, such as Asia and Latin America. In Mexico, approximately 2 billion pesos have been estimated to be spent in direct costs related to gastric cancer and breast cancer only during the first year of medical care. The WHO estimates that the number of patients affected by these neoplasms will double by $2020 .^{23}$

The main risk factor for this cancer is the epsilon proteobacterium Helicobacter pylori, which has an extraordinary ability to colonize the stomach of patients. Although in most cases the infection is asymptomatic, in some it is the cause of peptic ulcer and, in worst case scenario, of gastric cancer. The discovery that an infection was a risk factor for cancer has led to major changes in the management of modern medicine.

H. pylori infection global prevalence remains high, although it is declining in many regions of the world; however, in Asia and Latin America, 60 to $70 \%$ of adults have been estimated to be carriers of this pathogen. ${ }^{24}$ 
In Mexico, a seroprevalence study was carried out with more than 11 thousand sera from representative patients of all ages and from all states of the Mexican Republic. The infection was found to have its onset within the first years of life, as it occurs in developing countries, and after 20 years of age, more than $70 \%$ of adults are infected and remain with high infection rates throughout their lives. ${ }^{24}$

The reconstruction of $H$. pylori natural history indicates that during childhood this pathogen colonizes the gastric mucosa, where it establishes a signaling towards the epithelium, which responds with an inflammation reaction that causes gastritis, which in most cases is chronically established and can remain asymptomatic throughout the entire life. However, there is exaggerated inflammation of the gastric mucosa that can lead to atrophy when the bacteria have virulence genes and the epithelium shows higher sensitivity to the inflammatory response. If the inflammation and the bacterium that causes it remain, pre-neoplastic lesions can develop, which will eventually evolve to gastric cancer.

Solid evidence has been identified indicating that the infection can have a beneficial effect within the first years of life: epidemiological studies show an inverse association between $H$. pylori infection and adenocarcinoma of the esophagus. Apparently, when the bacterium is in the stomach, it acts as a biological buffer that prevents reflux and constant contact with acid in the esophagus, which ultimately leads to adenocarcinoma. ${ }^{25}$

H. pylori infection has also been found to be associated with a reduction in the risk for asthma and allergy, which is why this relationship with other autoimmune diseases is being studied. In Germany, newborn mice models showed that dendritic cell-produced interleukin 18 stimulates and differentiates regulatory $\mathrm{T}$ cells that inhibit autoimmune responses. Apparently, Helicobacter pylori stimulates regulatory $T$ cells in the stomach during childhood and helps to prevent autoimmune diseases. Due to the above observations, it is not certain whether $H$. pylori infection should be eradicated, since there are signs that this bacterium is part of stomach microbiota and has beneficial physiological functions; therefore, during the first years of infection there is no major harm. ${ }^{26}$ Precancerous lesions appear after 40 years of age, and actions should be taken before they manifest themselves in order to prevent the development of gastric cancer.

\section{Recommendations in the treatment of antimicrobial-resistant $H$. pylori}

International recommendations are mainly based on studies carried out in Europe and China; research in the United States is scarce, and in Canada there is no reliable information on resistance of this bacterium. ${ }^{27,28}$

In Mexico, clinical trials have been carried out with different therapeutic regimens. Triple standard therapy was tested in several thousand patients from seven places in Latin America: Mexico (Sonora and Chiapas), Honduras, Nicaragua, Costa Rica, Colombia and Chile. In the populations where the infection was eradicated, treatment for 14 days was the most effective and showed completely different results to those observed in China and Europe. Hence the importance of regional, local and national studies, in order to know the true efficacy of treatments in different populations. ${ }^{29}$

Taking into account the previous research and other investigations carried out by the Latin American Network for Antimicrobial Resistance Surveillance, in the Latin American 2014 consensus, triple standard therapy for 14 days is recommended as the first option, and as the second, the combination of omeprazole, amoxicillin and clarithromycin, where clarithromycin resistance defines the treatment usefulness. ${ }^{30}$

In Mexico, Helicobacter pylori resistance to antimicrobials has been studied for approximately 20 years. When the European consensus was established, it was below $5 \%$, and clarithromycin-specific resistance was $12 \%$, although this antibiotic was still clinically useful; resistance to metrodinazole was $40 \%$, although with variations; in the different periods examined, $7 \%$ resistance to amoxicillin was found. In the last collection of strains in $2017,60 \%$ resistance to metrodinazole was recorded, and resistance to clarithromycin was relevant. ${ }^{31}$

In 2018, the $4^{\text {th }}$ Mexican Consensus on Helicobacter pylori was established in collaboration with the Mexican Association Gastroenterology, where as a first line of treatment for regions with high rates of dual resistance (clarithromycin and metronidazole), the quadruple regimen is recommended for 14 days, with the possibility of considering two options: ${ }^{31}$

- Quadruple therapy with bismuth salts: proton pump inhibitor, bismuth subcitrate, tetracycline and metronidazole.

- Quadruple therapy without bismuth (concomitant therapy): bismuth subcitrate, amoxicillin, clarithromycin and metronidazole. 
In 2014, the WHO convened Helicobacter pylori experts for the development of recommendations for countries where gastric cancer is a serious problem. There is a proposal of basic screening to identify candidates for the bacterial eradication regimen, ${ }^{32}$ indicating that high-risk populations can be detected and age groups selected in a country. For example: in Mexico, the highest mortality rates due to gastric cancer are observed in Yucatán, Chiapas, Zacatecas and Baja California, states where it would be important for attention to be focused on.

Helicobacter pylori infections continue to be common in Mexican adults; $3 \%$ of them evolve to gastric cancer, with a high mortality rate and very high costs for the health system. Current Mexican consensus has corrected first-choice treatment regimen and eradication of the infection in adults older 40 than years without pre-neoplastic lesions is expected to be an effective measure to prevent the development of gastric cancer; however, this is something that remains to be proven.$^{30}$

\section{References}

1. World Health Organization. World Health Day. Suiza: WHO; 2011.

2. World Health Organization. La resistencia a los antimicrobianos. Suiza: WHO; 2019.

3. World Health Organization. Farmacorresistencia. Suiza: WHO; 2015.

4. World Health Organization. Drug resistance. Suiza: WHO; 2015.

5. Centers for Disease Control and Prevention. National Healthcare Safety Network. EE. UU.: CDC; 2019

6. Mussaret BZ, Dreser A, Figueroa IM. A collaborative initiative for the containment of antimicrobial resistece in Mexico. Zooneses Public Health. $2015 ; 62: 52-57$.

7. Acuerdo por el que se declara la obligatoriedad de la Estrategia Nacional de Acción contra la Resistencia a los Antimicrobianos. México: Diario Oficial de la Federación; 2018 Jun 5.

8. Red Latinoamericana de Vigilancia de la Resistencia a los Antimicrobianos (ReLAVRA). EE. UU.: Organización Panamericana de la Salud; 2019.

9. Universidad Nacional Autónoma de México. Plan Universitario de Control de la Resistencia Antimicrobiana. México: UNAM; 2018.

10. Garza-González E, Morfín-Otero R, Mendoza-Olazarán S, Bocanegra-Ibarias $P$, Flores-Treviño $S$, Rodríguez-Noriega $E$, et al. A snapshot of antimicrobial resistance in Mexico. Results from 47 centers from 20 states during a six-month period. PLoS One. 2019;14:e0209865.
11. Universidad de Colima [sitio web]. Red Temática de Investigación y Vigilancia de la Farmacorresistencia. México: Universidad de Colima. [Actualización: 2019].

12. O'Neill CB. The review on antimicrobial resistence. Suiza: World Intellectual Property Organization; 2016.

13. Centers for Disease Control and Prevention. Vital signs. EE. UU.: CDC; 2018.

14. Walsh CT, Wencewicz TA. Prospects for new antibiotics: a molecule-centeres perspective. J Antibiot (Tokyo). 2014;67:7-22.

15. World Health Organization. Resistencia a los antimicrobianos. Suiza: WHO; 2016.

16. Codjoe FS, Donkor ES. Carbapenem resistance: a review. Med Sci. 2017;6:1-21.

17. Köck R, Daniels-Haardt I, Becker K, Mellman A, Friedrich A, Mevius D, et al. Carbapenem-resistant Enterobacteriaceae in wildlife, food-producing, and companion animals: a systematic review. Clin Microbiol Infect. 2018;24:1241-1250.

18. Wernli D, Jørgensen PS, Harbarth S, Carroll SP, Laxminarayan R, Levrat $\mathrm{N}$, et al. Antimicrobial resistance: the complex challenge of measurement to inform policy and the public. PLoS Med. 2017;14:e1002378.

19. Santajit S, Idrawattana N. Mechanisms of antimicrobial resistance in ESKAPE pathogens. BioMed Res Int. 2016;2475067:2016

20. Llaca-Díaz JM, Mendoza-Olazarán S, Camacho-Ortiz A, Flores S, Garza-González E. One-year surveillance of ESKAPE pathogens in an Intensive Care Unit of Monterrey, Mexico. Chemotherapy. 2012;58:475-481.

21. Velázquez-Acosta $C$, Cornejo-Juárez $P$, Volkow-Fernández $P$. Cepas E-ESKAPE multidrogorresistentes aisladas en hemocultivos de pacientes con cáncer. Salud Publica Mex. 2015;60:151-157.

22. Yoon H, Kim N. Diagnosis and management of high risk group for gastric cancer. Gut and Liver. 2015;9:5-17.

23. Ferlay J, Colombet M, Bray F. Cancer Incidence in five continents. Francia: International Agency for Research on Cancer; 2019.

24. Rawla P, Barsouk A. Epidemiology of gastric cancer: global trends, risk actors and prevention. Prz Gastroenterol. 2019;14:26-38.

25. Román-Román A, Martínez-Carrillo DN, Atrisco-Morales J, Azúcar-Heziquio JC, Cuevas-Caballero AS, Castañón-Sánchez CA, et al. Helicobacter pylori vacA s1m1 genotype but not cagA or babA2 increase the risk of ulcer and gastric cancer in patients from Southern Mexico. Gut Pathog. 2017;9:18.

26. Burkitt MD, Duckworth CA, Williams JM, Pritchard DM. Helicobacter pylori-induces gastric pathology: insights from in vivo and ex vivo models. Dis Model Mech. 2017;10:89-104.

27. Kariya, S, Okano M, Nishizaki K. An association between Helicobacter pylori and upper respiratory tract disease: fact or fiction? World J Gastroenterol. 2014;20:1470-1484.

28. Hu Y, Zhu Y, Lu NH. Novel and effective therapeutic regimens for Helicobacter pylori in an era of increasing antibiotic resistance. Front Cell Infect Microbiol. 2017:7:168.

29. Porras C, Nodora J, Sexton R, Ferreccio C, Jiménez S, Domínguez RL, et al. Epidemiology of Helicobacter pylori infection in six Latin American countries (SWOG Trial S0701). Cancer Causes Control. 2013;24:209-215.

30. Garza-González E, Pérez-Pérez GI, Maldonado-Garza HJ, Bosques-Padilla FJ. A review of Helicobacter pylori diagnosis, treatment, and methods to detect eradication. World J Gastroenterol. 2014;20:1438-1449.

31. Bosques-Padilla FJ, Remes-Troche JM, González-Huezo MS, Pérez-Pérez G, Torres-López J, Abdo-Francis JM, et al. IV Consenso Mexicano sobre Helicobacter pylori. Rev Gastroenterol Mex. 2018;83(3):325-341.

32. Mascellino MT, Porowska B, de Angelis M, Oliva A. Antibiotic susceptibility, heteroresistance, and updated treatment strategies in Helicobacter pylori infection. Drug Des Devel Ther. 2017;11:2209-2220. 